\title{
Nanocompósitos de Polipropileno e Argila Organofílica: Difração de Raio X, Espectroscopia de Absorção na Região do Infravermelho e Permeação ao Vapor D’água
}

\author{
Fernanda C. Morelli, Adhemar Ruvolo Filho \\ Departamento de Química, UFSCar
}

\begin{abstract}
Resumo: Neste trabalho foram preparados nanocompósitos de polipropileno, polipropileno graftizado com anidrido maleico como agente compatibilizante e argila organofílica comercial (montmorilonita) de codinome Cloisite $20 \mathrm{~A}$ em concentrações de 1,5, 2,5, 5,0 e 7,5\% por meio da técnica de intercalação no estado fundido utilizando extrusora de dupla rosca. Os materiais obtidos foram caracterizados por difração de raio X, espectroscopia de absorção na região do infravermelho com transformada de Fourier e análise da permeação ao vapor d'água. Os resultados de difração de raio X e da espectroscopia de absorção na região do infravermelho indicaram a formação de nanocompósitos com estruturas provavelmente esfoliadas e/ou intercaladas para as concentrações de 1,5 e 2,5\% de argila organofílica, e proporcionaram uma acentuada diminuição da permeabilidade, corroborando com as demais análises.
\end{abstract}

Palavras-chave: Nanocompósitos, polipropileno, permeação de vapor de água, montmorilonita.

\section{Nanocomposites of Polypropylene and Organophilic Clay: X Ray Diffraction, Absorption Infrared Spectroscopy with Fourier Transform and Water Vapor Permeation}

\begin{abstract}
In this work nanocomposites were prepared from polypropylene, graft polypropylene with maleic anhydride as compatibilizer and organophilic montmorillonite Cloisite 20A with concentrations of 1.5, 2.5, 5.0 and 7.5\% clay. The mixture was made in the melt state using a twin screw extruder. The materials were characterized by $\mathrm{X}$ ray diffraction, infrared spectroscopy with Fourier transform and analysis of water vapor permeation. The results of X ray diffraction and absorption infrared spectroscopy indicates the formation of nanocomposites with structures probably exfoliate and / or intercalated for concentrations of 1.5 and $2.5 \%$ clay, and provided a marked decrease in the water permeability, corroborating with other analyses.
\end{abstract}

Keywords: Nanocomposites, polypropylene, water vapor permeability, montmorillonite clay.

\section{Introdução}

A mistura de polímeros e argilas organofílicas tem sido muito estudada nas últimas décadas para a produção de nanocompósitos. Os nanocompósitos constituem uma classe de materiais formados por híbridos orgânicos e inorgânicos, nos quais sua fase inorgânica está dispersa em nível nanométrico. Essa classe de materiais começou a ser estudada na década de 80 pelo laboratório de Pesquisa da Toyota com o desenvolvimento de nanocompósitos de poliamida e $\operatorname{argila}^{[1,2,3]}$.

O nanocompósito estudado nesse trabalho é conhecido como polímero de silicato em lamelas (PLS). A fase inorgânica (argila) dessenanocompósitoencontra-se dispersa e sob a forma de camadas (lamelas) de aproximadamente um nanômetro de espessura e centenas de nanômetros de largura e comprimento, possuindo, portanto um elevado fator de forma ${ }^{[1,4]}$.

A obtenção de nanocompósitos tem atraído muito o interesse de pesquisadores ${ }^{[5,6]}$, pois nanocompósitos baseados na inserção de nanoentidades, na forma de argilominerais em matrizes poliméricas, foram capazes de gerar materiais com elevadas propriedades mecânicas, de barreira (reduzida difusão de espécies gasosas, por exemplo), elevadas propriedades térmicas, entre outras.

Com relação às propriedades de barreira, a redução da permeabilidade em relação à matriz polimérica pura tem sido atribuída ao elevado fator de forma das camadas de argilo-mineral, que aumentam o caminho livre percorrido por um determinado penetrante no material, e a restrição imposta pela baixa mobilidade das cadeias poliméricas entre os espaços lamelares do argilo-mineral.

Um dos métodos de processamento de nanocompósito é o de intercalação no estado fundido. Neste caso, o polímero é misturado com a argila organofílica no estado fundido de forma a permitir a intercalação das cadeias do polímero entre as lamelas da argila. Devido à elevada queda de entropia conformacional das cadeias poliméricas intercaladas, a força motriz desse processo é a contribuição entálpica 
das interações polímero/argila durante o processamento. As tensões cisalhantes, ao promoverem a destruição dos aglomerados de argila e o acesso das cadeias poliméricas às partículas primárias da argila, auxiliam o processo de intercalação, permitindo sua ocorrência em sistemas onde há uma difícil dispersão entre a matriz polimérica e a $\operatorname{argila}^{[7]}$.

A literatura dos últimos dez anos ${ }^{[1,6]}$ tem mostrado resultados significativos em termos de propriedades mecânicas e de barreira de nanocompósitos de argilomineral em matrizes de nylon, poli (tereftalato de etileno), policarbonato entre outros. No entanto, nos trabalhos reportados sobre nanocompósitos de matrizes olefínicas ${ }^{[8]} \mathrm{o}$ que se observa é que a compatibilidade em termos de adesão interfacial polímero/argila ainda é um desafio em aberto, fato comprovado pela pouca influência do argilo-mineral nas propriedades mecânicas destes materiais, assim como na dificuldade para se obter um bom nível de esfoliação do argilo-mineral.

Este trabalho descreve a preparação de nanocompósito de polipropileno/polipropileno graftizado com anidrido maleico e argila montmorilonita organofílica a partir da mistura no estado fundido e a caracterização estrutural por difração de raio X e espectroscopia na região do infravermelho com transformada de Fourier e a caracterização da propriedade de barreira utilizando vapor de água.

\section{Experimental}

\section{Materiais}

Os materiais utilizados na preparação dos nanocompósitos foram o polipropileno PP H501C da empresa Braskem - Brasil $\left(\mathrm{MFI}=3,5 \mathrm{~g} / 10\right.$ minutos, a $230{ }^{\circ} \mathrm{C}$ sob carga de $2,16 \mathrm{~kg}$ ), o compatibilizante polipropileno graftizado com anidrido maleico (PP-g-AM) OREVAC CA-100 da empresa Arkema - Brasil $\left(\right.$ MFI $=150-200 \mathrm{~g} / 10$ minutos, a $230{ }^{\circ} \mathrm{C}$ sob carga de $2,16 \mathrm{~kg}$ ) e a argila montmorilonita organofílica de codinome Cloisite 20A fornecida pela empresa Southern Clay Company - Estados Unidos.

\section{Métodos}

A preparação dos nanocompósitos foi realizada em duas etapas. A primeira consistiu de uma mistura de PP-g-AM e argila, na proporção 1:3 em peso, utilizando-se um misturador com alta taxa de cisalhamento $(\mathrm{w}=3000 \mathrm{rpm})$ produzindo um concentrado com $25 \%$ de argila devido à proporção utilizada. A segunda etapa consistiu na moagem criogênica do concentrado utilizando um moinho modelo IKA Werke M20 da marca Merse e em seguida a diluição do concentrado na matriz de PP, para obtenção dos nanocompósitos via intercalação no estado fundido fazendo uso de uma extrusora com dupla rosca modelo MT19TC-25:1 da marca B \& P Process Equipament and Systems, $\mathrm{L} / \mathrm{D}=25$, com o seguinte perfil de temperaturas: $187^{\circ} \mathrm{C} / 200^{\circ} \mathrm{C} / 210^{\circ} \mathrm{C} / 210^{\circ} \mathrm{C} / 210^{\circ} \mathrm{C}$ e velocidade de rotação das roscas de $150 \mathrm{rpm}$. As amostras
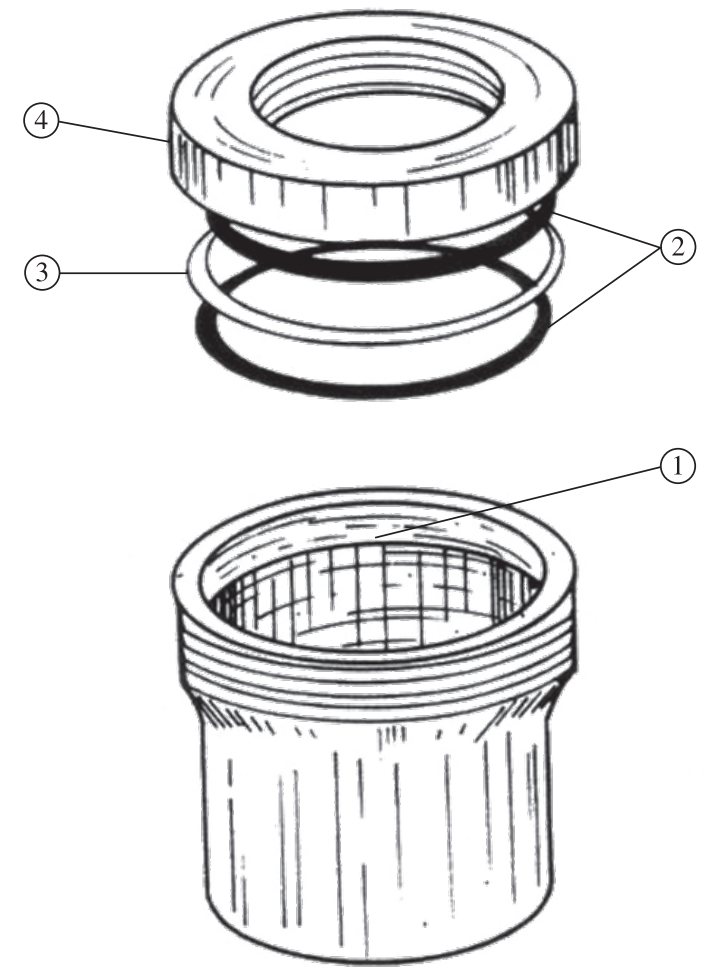

Figura 1. Célula de pesagem (Copo de Payne) para as medidas de transporte de vapor d'água. 1) borda interna para selagem do filme polimérico; 2) anéis de borracha; 3) anel de alumínio; 4) tampa do copo ${ }^{[9]}$.

foram obtidas com as seguintes concentrações de argila organofílica: 1,5, 2,5, 5,0 e 7,5\%. Em seguida, para realização das análises, a partir de cada concentração de argila organofílica foram preparadas amostras na forma de filmes, via termoprensagem na temperatura de $180{ }^{\circ} \mathrm{C}$, seguido de resfriamento sob pressão até a temperatura ambiente.

A análise estrutural foi realizada por difração de raio $\mathrm{X}$ (DRX) utilizando o equipamento da marca Rigaku, modelo Geigerflex, com varredura $2 \theta$ na faixa de $2^{\circ}$ a $10^{\circ}$, com radiação $\mathrm{CuK} \alpha 1,54 \AA$, potência no tubo de $40 \mathrm{kV}$ e corrente de $25 \mathrm{~mA}$.

A análise termogravimétrica foi realizada para calcular a porcentagem real de argila pura presente nas amostras de PP/PP-g-AM/argila organofílica utilizando um módulo termogravimétrico TA Q500 da TA Instruments e as condições empregadas foram: aquecimento de $30-800{ }^{\circ} \mathrm{C}$, com uma taxa de aquecimento de $10^{\circ} \mathrm{C} / \mathrm{min}$ sob atmosfera inerte de nitrogênio $\left(\mathrm{N}_{2}\right)$ com vazão de gás de $100 \mathrm{~mL} / \mathrm{min}$.

As análises de espectroscopia de absorção na região do infravermelho com transformada de Fourier (FTIR) foram realizadas em um equipamento Bomem Hartmann \& Braun/MB modelo 102 seguindo as seguintes condições de operação: região $400-4000 \mathrm{~cm}^{-1}$, com 32 varreduras e resolução de $2 \mathrm{~cm}^{-1}$.

Para a realização da análise de permeação ao vapor de água montou-se um sistema ${ }^{[9]}$, representado pela Figura 1, no qual se pode analisar a capacidade de permeação de cada amostra por meio de sua perda de massa (que é neste caso 
diretamente proporcional à perda de água que flui através do filme). Através do fluxo (J) determinado pela variação temporal da massa corrigida pela área do filme exposta (Equação 1):

$$
\left(\frac{\delta m}{\delta T}\right) \quad \frac{1}{\mathrm{~A}}=\mathrm{J}
$$

Com uso da $1^{\text {a }}$ Lei de Fick, pode-se determinar assim o coeficiente de permeabilidade ao vapor de água $\left(\mathrm{P}_{\mathrm{w}}\right)$, conhecendo-se o gradiente de pressão de vapor de água $(\Delta \mathrm{P})$, e a espessura da amostra (L) (Equação 2). Todos os ensaios foram realizados na temperatura de $25^{\circ} \mathrm{C}$.

$$
J=\frac{P_{w} \Delta P}{L}
$$

\section{Resultados e Discussão}

Na Tabela 1 estão apresentados os resultados de TGA, para a quantificação da quantidade real de material inorgânico, ou seja, argila pura presente nas amostras estudadas (Nano 1,5, 2,5, 5,0 e 7,5\% de argila organofílica), tendo-se em conta que a argila organofílica (Cloisite 20A) na sua composição tem $70 \%$ de argila pura e 30\% de modificador orgânico [sal de amônio quaternário formado por dois grupos metila e duas cadeias carbônicas dihidrogenadas (HT)].

Com base na quantidade de resíduos inorgânicos (argila pura) encontrado a $600{ }^{\circ} \mathrm{C}$, pode-se observar que houve uma pequena perda da porcentagem originalmente adicionada da argila organofílica, tanto no concentrado PP-G-MA/argila organofílica como em cada nanocompósito, o que é comum durante as etapas de mistura e extrusão.

A Figura 2 apresenta o conjunto típico de curvas termogravimétricas obtidos para os nanocompósitos preparados neste trabalho.

Na Figura 3 estão apresentados os padrões de DRX obtidos para a argila organofílica e para as amostras dos nanocompósitos. Pode-se observar que a argila organofílica apresentou um pico de difração em $2 \theta$ em torno de $3,57^{\circ}$, que corresponde ao espaçamento basal de $2,47 \mathrm{~nm}$ do plano difratográfico 001 , e um segundo pico em $2 \theta$ a $7,28^{\circ}$, que corresponde ao espaçamento basal de $1,21 \mathrm{~nm}$ do plano difratográfico 002. Para as amostras dos nanocompósitos,

Tabela 1. Valores obtidos das \% de resíduos inorgânicos (argila pura) a $600^{\circ} \mathrm{C}$ por TGA para a amostra de PP-G-MA/Argila organofílica, PP extrudado e para PP/PP-g-AM/argila organofílica com diferentes concentrações de argila organofílica.

\begin{tabular}{ccc}
\hline Amostra & $\begin{array}{c}\text { \% resíduo } \\
\mathbf{a ~ 6 0 0}{ }^{\circ} \mathbf{C}\end{array}$ & $\begin{array}{c}\text { \% teórica de argila } \\
\text { na mistura original }\end{array}$ \\
\hline PP-g-MA/argila organofílica & 15,56 & 17,50 \\
Pp extrudado & 0 & 0 \\
Nano 1,5\% & 1,13 & 1,05 \\
Nano 2,5\% & 1,72 & 1,75 \\
Nano 5,0\% & 2,91 & 3,5 \\
Nano 7,5\% & 4,47 & 5,25 \\
\hline
\end{tabular}

os picos da argila sofreram um ligeiro descolamento para valores menores em $2 \theta$, ou seja, na presença do polímero e do compatibilizante, para todas as concentrações das amostras, o pico principal em $2 \theta$ em torno de $3,57^{\circ}$ da argila aparece em $2 \theta$ em torno de $2,90^{\circ}$ que corresponde a um espaçamento basal de 3,04 nm. Nota-se também que para as amostras de concentrações correspondentes a 5,0 e 7,5\% de argila organofílica houve uma acentuada diminuição de suas intensidades e para as concentrações de 1,5 e 2,5\% de argila organofílica os picos praticamente desaparecem. Esse decaimento da intensidade do pico principal da argila pura em relação às amostras sugere uma desorganização das lamelas da argila presente nas amostras de nanocompósitos. Esses fatos indicam uma provável formação de nanocompósitos de estruturas intercaladas e/ou esfoliadas para as amostras de 1,5 e $2,5 \%$ de argila organofílica.

Na Figura 4 estão apresentados os resultados de FTIR para as amostras dos nanocompósitos, na região entre 1650 a $1900 \mathrm{~cm}^{-1}$. Nesta região espectral podem-se observar as bandas típicas do anidrido maleico nas amostras de nanocompósitos. As bandas de absorção com picos em 1785 e $1830 \mathrm{~cm}^{-1}$ estão associadas respectivamente ao estiramento

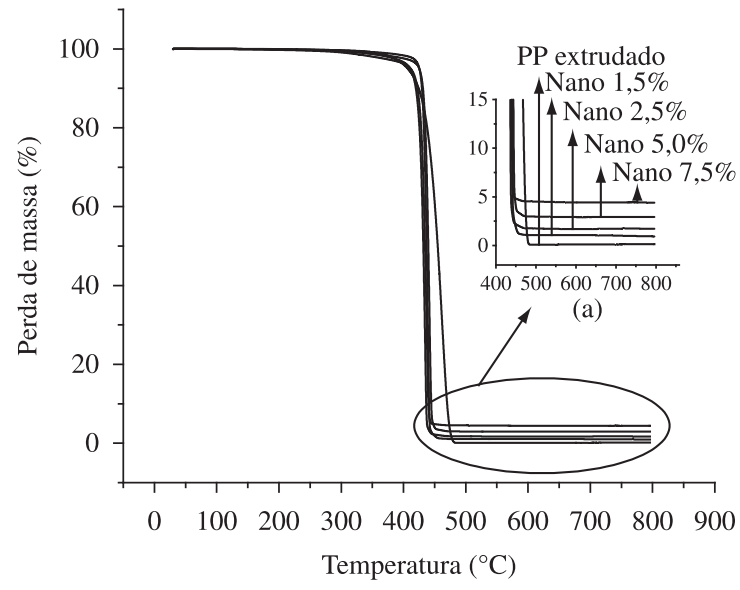

Figura 2. Curvas de TGA comparativas para o PP extrudado e para as amostras de PP/PP-g-AM/argila organofílica em diferentes concentrações de argila organofílica com ampliação na região de $400-900{ }^{\circ} \mathrm{C}$ caracterizada pelo gráfico a).

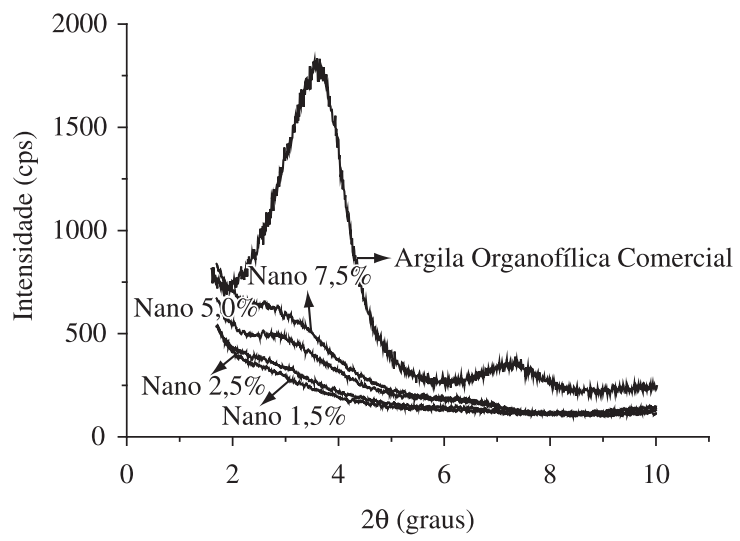

Figura 3. Padrões de difração de raio X para as amostras de PP/PP-g-AM/ argila organofílica em diversas concentrações de argila organofílica. 


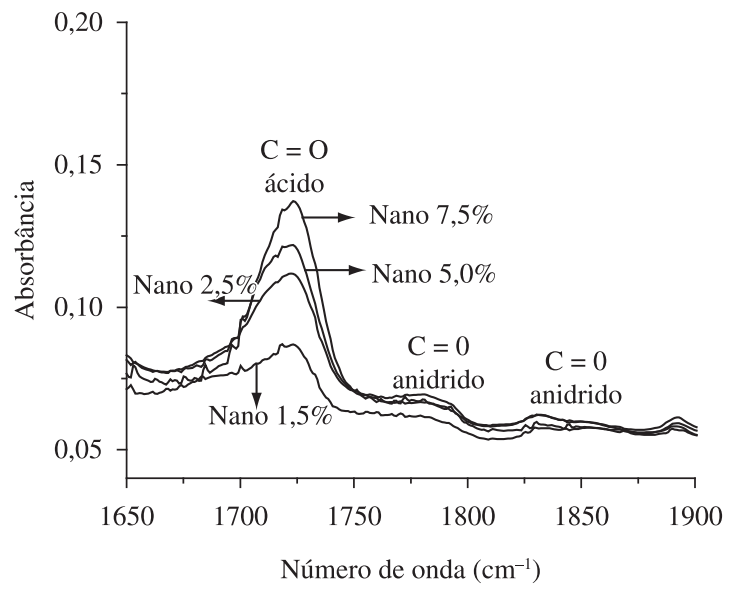

Figura 4. Espectros de absorção na região do infravermelho, ampliado na região de $1650-1900 \mathrm{~cm}^{-1}$, para as amostras de nanocompósitos com diferentes concentrações de argila organofílica.

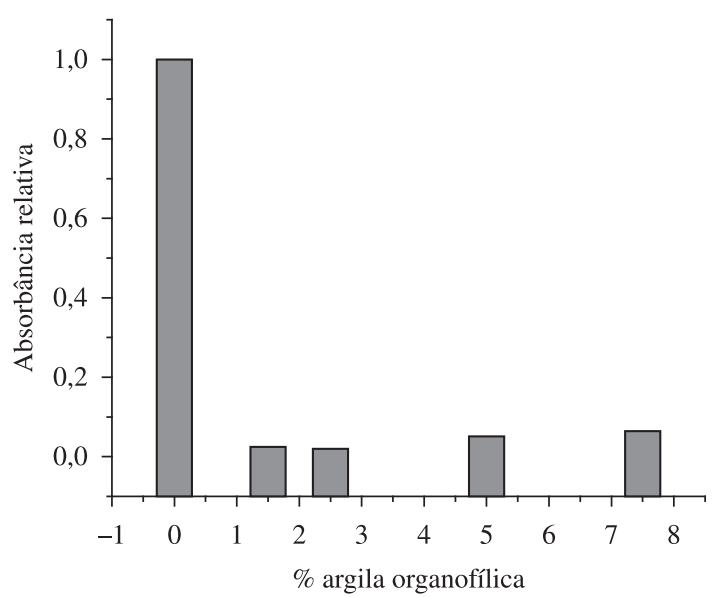

Figura 5. Absorbância relativa $\left\{\left[\mathrm{A}_{1785} / \mathrm{A}_{1167}\right]_{\text {Nano }} /\left[\mathrm{A}_{1785} / \mathrm{A}_{1167}\right]_{\mathrm{PP}-\mathrm{g}-\mathrm{AM}}\right\}$ em função da porcentagem de argila organofílica para as amostras de nanocompósitos PP/PP-g-AM/argila organofílica com diferentes teores de argila organofílica mostrando a interação do anidrido maleico na superfície da argila.

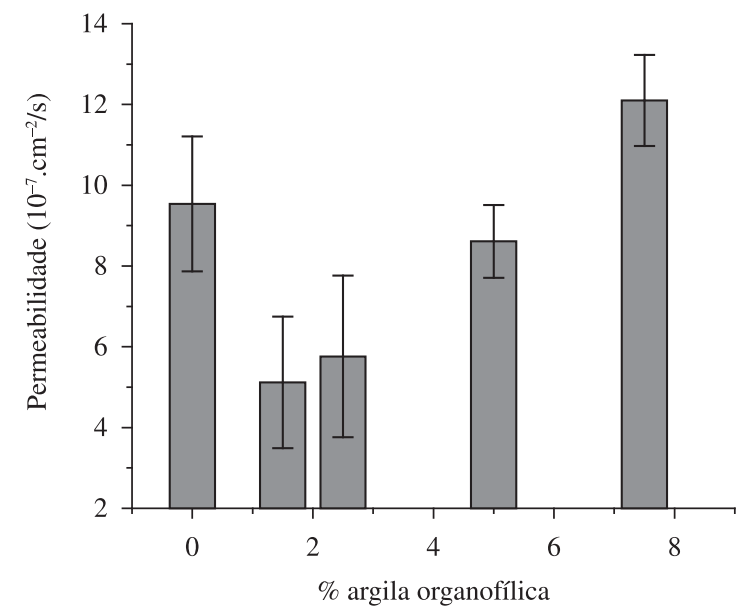

Figura 6. Permeabilidade em função da porcentagem de argila organofílica nas amostras de PP extrudado e PP/PP-g-AM/argila organofílica com diferentes teores de argila organofílica. simétrico e assimétrico da carbonila do anidrido e a banda de absorção com pico em $1730 \mathrm{~cm}^{-1}$ é normalmente associada à carbonila do ácido maleico ${ }^{[10]}$.

Uma avaliação qualitativa da interação entre os grupos anidrido do agente de compatibilização (PP-g-AM) e a superfície das lamelas da argila foi realizada através da área da banda de absorção com pico em $1784 \mathrm{~cm}^{-1}$ utilizando-se o método de deconvolução através de uma função de Lorentz. Como banda interna de referência utilizou-se o pico de absorção no comprimento de onda de $1167 \mathrm{~cm}^{-1}$ atribuída à deformação assimétrica dos grupos metila da cadeia do PP. Uma redução na área de absorção dos grupos carbonila do anel de cinco átomos de carbono do grupo anidrido, tendo como referência o PP-g-AM puro, é um indicativo da interação do compatibilizante com a superfície das lamelas da argila. A fixação destes grupos na superfície da argila diminui a quantidade de grupos carbonila livres para interação com a radiação infravermelha. Os resultados obtidos, da absorbância relativa $\left\{\left[\mathrm{A}_{1785} / \mathrm{A}_{1167}\right]_{\text {Nano }} /\left[\mathrm{A}_{1785} / \mathrm{A}_{1167}\right]_{\mathrm{PP}-\mathrm{g}-\mathrm{AM}}\right\}$ em função da porcentagem de argila organofílica, estão apresentados na Figura 5 e indicam que está ocorrendo interação do anidrido com a argila para todas as concentrações estudadas, no entanto, com maior relevância, nos nanocompósitos com concentração de argila organofílica de 1,5 e 2,5\%, corroborando com os padrões de DRX, apresentados na Figura 3.

Na Figura 6 estão apresentados os resultados obtidos da permeabilidade ao vapor de água onde se observa que para os nanocompósitos com concentrações de argila organofílica de 1,5 e 2,5\% houve uma redução expressiva na permeabilidade, em relação ao PP extrudado, na faixa de 46 e $40 \%$ respectivamente. As amostras de 5,0 e 7,5\% de argila organofílica não mostraram nenhuma melhoria em relação a sua permeabilidade. Estes resultados corroboram com os padrões de DRX, apresentados na Figura 3, principalmente para as concentrações de 1,5 e 2,5\% de argila organofílica.

\section{Conclusões}

- A seqüência de operações adotada para o processamento dos nanocompósitos parece ser adequada para a intercalação, em todas as concentrações estudadas, e talvez para a esfoliação da argila, principalmente nas concentrações de 1,5 e 2,5\% de argila organofílica;

- A confirmação da intercalação e esfoliação da argila nas concentrações de 1,5 e 2,5\% de argila organofílica será um resultado inédito, pois a literatura, de forma geral, tem mostrado resultados equivalentes somente para concentrações maiores ou iguais a 5\% de argila organofílica;

- A redução na permeabilidade ao vapor d'água, na ordem de 46-40\%, para os nanocompósitos com concentração de argila organofílica de 1,5 e 2,5\%, respectivamente, é um resultado expressivo tendo-se em conta uma possível prospecção para a aplicação deste material na área de filmes. 


\section{Agradecimentos}

A CAPES pela bolsa de mestrado e ao CNPq e Fapesp pelo apoio direto ou indireto para a realização do projeto.

\section{Referências Bibliográficas}

1. Alexandre, M. \& Dubois, P. - Mater. Sci. Eng., 28, p. 1-63 (2000).

2. Rodrigues, A.; Brasileiro, M. I.; Araujo, W. D.; Araujo, E. M.; Neves, G. A. \& Melo, T. J. A. - Polímeros, 17, p. 219 (2007).

3. Pavlidou, S. \& Papaspyrides, S. D. - Progr. Polymer Sci., 33, p. 1119 (2008).

4. Paiva, L. B.; Morales, A. R. \& Guimarães, T. R. Polímeros, 16, p. 136 (2006).

5. Okamoto, M. \& Ray, S. S. - Progr. Polymer Sci., 28, p. 1539 (2003).
6. Kornmann, X. - "Synthesis and characterization of thermoset-layered silicate nanocomposites", $\mathrm{PhD}$. Thesis, Luleå Tekniska Universitet, Sweden (2000).

7. Hasegawa, N.; Kawasumi, M.; Kato, M. \& Usuki, M. J. Appl. Polymer Sci., 67, p. 87 (1998).

8. Osman, M.; Rupp, J. E. P. \& Suter, U. W. - Polymer, 46, p. 1653 (2005).

9. Sampaio, E. M. V. - "Morfologia de filmes de poliestirenos comerciais, produzidos por "casting" a partir de solução e termoprensagem”, Master Thesis, Universidade Federal de São Carlos, São Carlos (1993).

10. Carvalho, G. M. X.; Mansur, H. S.; Vasconcelos, W. L. \& Orefice, R. L. - Polímeros, 17, p. 98 (2007).

Enviado: 30/09/09

Reenviado: $18 / 12 / 09$

Aceito: $26 / 01 / 10$

DOI: $10.1590 / \mathrm{S} 0104-14282010005000014$ 